\title{
Properties of prominence fine-structure threads derived from SOHO/SUMER hydrogen Lyman lines
}

\author{
S. Gunár ${ }^{1,2,3}$, P. Heinzel ${ }^{1,2}$, B. Schmieder ${ }^{4}$, P. Schwartz ${ }^{1}$, and U. Anzer ${ }^{2}$ \\ 1 Astronomical Institute, Academy of Sciences of the Czech Republic, 25165 Ondřejov, Czech Republic \\ e-mail: [gunar;pheinzel] @asu.cas.cz \\ 2 Max-Planck-Institut für Astrophysik, Karl-Schwarzschild-Strasse 1, 85740 Garching, Germany \\ 3 Astronomical Institute, Faculty of Mathematics and Physics, Charles University, Prague, Czech Republic \\ 4 Observatoire de Paris, Section de Meudon, 92195 Meudon Principal Cedex, France
}

Received 3 May 2007 / Accepted 8 June 2007

\section{ABSTRACT}

\begin{abstract}
Context. The SOHO/SUMER observations provide us for the first time with the prominence spectra in the Lyman- $\alpha$ line outside the attenuator together with the higher members of the hydrogen Lyman series.

Aims. We derive the prominence fine-structure thread properties by comparing the SOHO/SUMER hydrogen Lyman series observations with the synthetic Lyman lines.

Methods. To obtain the synthetic profiles of the Lyman lines, we used 2D prominence fine-structure thread models with a PCTR and consistently solved the 2D non-LTE multilevel radiative transfer. The trial-and-error method was applied to find the model with the best agreement between the synthetic Lyman line profiles and the observed ones.

Results. The properties of the resulting model with the best match of the synthetic and observed line profiles are central (minimum) temperature $T_{0}=7000 \mathrm{~K}$, maximum column mass in the centre of the thread $M_{0}=1.1 \times 10^{-4} \mathrm{~g} \mathrm{~cm}^{-2}$, horizontal field strength in the middle of the thread $B_{x}(0)=6$ Gauss and the boundary pressure $p_{0}=0.015 \mathrm{dyn} \mathrm{cm}^{-2}$.

Conclusions. The Lyman line profiles observed by SOHO/SUMER can be better reproduced by using multi-thread models consisting of a set of the $2 \mathrm{D}$ prominence fine-structure threads placed perpendicularly to the line-of-sight, rather than with the single-thread model.
\end{abstract}

Key words. Sun: prominences - radiative transfer - line: profiles

\section{Introduction}

The observations in the hydrogen Lyman lines and in the continuum, represent an important contribution to the understanding of the structure and physical properties of solar prominences. A considerable amount of spectral data in the Lyman lines for quiescent prominences is provided by the SUMER UV-spectrograph (Solar Ultraviolet Measurements of Emitted Radiation - Wilhelm et al. 1995) on board the SOHO (SOlar and Heliospheric Observatory). Some of these data and their analysis can be found in Schmieder et al. (1998, 1999, 2003, 2007), Heinzel et al. (2001, 2006), Gunár et al. (2006, 2007b) and in a review of SOHO prominence observations by Patsourakos \& Vial (2002). Prominence fine structures were recently reviewed by Heinzel (2007).

The formation depths of the Lyman lines and their individual parts (centre, peaks and wings) span the whole structure of the prominence from the centre to the prominence-corona transition region (PCTR). Thus their observations represent a significant constraint for prominence modelling. The proper interpretation of the Lyman line observations requires highly sophisticated models with complex non-LTE radiative transfer computations with the PCTR included. Heasley \& Mihalas (1976) constructed 1D prominence models in magnetohydrostatic (MHS) equilibrium of the Kippenhahn-Schlüter-type (Kippenhahn \& Schlüter 1957 ) with multi-level non-LTE radiative transfer. The importance of the PCTR for the prominence modelling has been shown by Anzer \& Heinzel (1999) and more recently by Labrosse \& Gouttebroze (2004). The latter authors concluded that the presence of the PCTR will be required to match the observations with their radiative transfer calculations of the $\mathrm{He}$ I triplet lines in a 1D vertical slab model. Heinzel \& Anzer (2001) presented prominence fine-structure models in 2D MHS equilibrium (with a PCTR) and consistently solved 2D radiative transfer (for further details see also Heinzel \& Anzer 2005). The importance of the 2D modelling for proper interpretation of the SUMER prominence observations was suggested by Heinzel et al. (2001).

A detailed analysis of the observed spectra of two quiescent prominences shows in one case strong unreversed emission profiles of the Lyman lines. The other prominence with a similar brightness in the Lyman spectrum exhibits a significant self-reversal in all observed lines. In order to explain such behaviour, one has to consider different orientations of the magnetic field lines with respect to the line-of-sight. If the prominence fine structure threads are seen from different sides with different shapes of the PCTR this can lead to the observed behaviour. Heinzel et al. (2005) have shown that the Lyman-line profiles are more reversed when seen across the field lines, compared to those seen along the magnetic field. This study, together with Gunár et al. (2007a) devoted to the Lyman continuum, represents an important step towards the understanding of the formation of the Lyman spectrum within solar prominences.

In the present paper we use the same 2D-modelling technique for analysing the SOHO/SUMER observations of the hydrogen Lyman-line series. We were able for the first time to analyse the Lyman- $\alpha$ line together with the higher members of the series. The previous study of the observed Lyman lines 


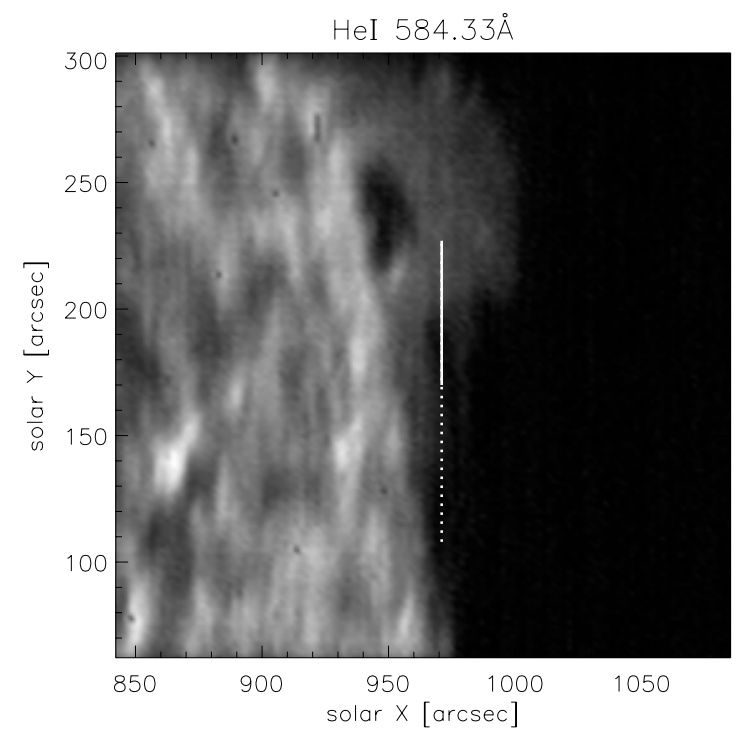

Fig. 1. CDS He I (584.33 $\AA$ ) line raster with indication of the SUMER slit centred at $X=972^{\prime \prime}$ and $Y=168^{\prime \prime}$ in SOHO coordinates. The full line represents the part of the SUMER slit which corresponds to the part of the detector A which is still operational.

(Gunár et al. 2007b) was limited to the Lyman- $\beta$ to Lyman- $\delta$ lines. Other studies concerning the analysis of the SOHO/SUMER observations with the usage of the same $2 \mathrm{D}$ modelling are the following: Gunár et al. (2006) devoted to the study of the Lyman- $\alpha$ raster and Schmieder et al. (2007) with a qualitative analysis of the Lyman- $\delta$ raster. The opportunity to study the Lyman series observations including the Lyman- $\alpha$, represents another important step towards the understanding of the physics of the fine-structure prominences.

The paper is organized as follows. Section 2 gives information on the observed prominence, the SUMER spectra and their calibration. Section 3 describes our 2D prominence finestructure thread model. Section 4 gives the comparison of the observed and synthetic spectra obtained by either single-thread or multi-thread modelling. Finally, Sect. 5 presents the discussion and our conclusions.

\section{Observations}

The SUMER instrument is a stigmatic spectrograph equipped with two photon-counting detectors ( $\mathrm{A}$ and $\mathrm{B}$ ). Both detectors have 1024 spectral columns and 360 spatial rows. Since May 2004 the detector A has been showing a deterioration of the electronics responsible for the readout in the $y$ direction ( $y$-ADC), affecting the spatial (along the slit) information, while the $x$-ADC is working correctly, leading to correct spectral information.

The present study is based on the spectroscopic observations of the quiescent prominence on May 25, 2005 carried out by SUMER (detector A) during the MEDOC coordinated campaign between SOHO and ground-based instruments (MEDOC campaign No. 15, May 16-29, 2005). The SUMER slit was pointed at solar coordinates $X=972^{\prime \prime}$ and $Y=168^{\prime \prime}$. The narrower slit $8\left(0.3^{\prime \prime} \times 120^{\prime \prime}\right)$ was used for Lyman- $\alpha$ observations, while for other Lyman lines slit $5\left(1^{\prime \prime} \times 120^{\prime \prime}\right)$ was used. Figure 1 shows the prominence in the He I (584.33 $\AA$ ) line observed by CDS (Coronal Diagnostic Spectrometer - Harrison et al. 1995) on SOHO with the position of the SUMER slit. The full line represents the part of the SUMER slit which corresponds to the part of the detector A which is still operational.

The Lyman series spectral data used in our study were obtained in time steps of approximately two minutes. Lyman- $\alpha$ observations began at 19:10:03 UT, Lyman- $\beta$ at 19:12:23 UT, Lyman- $\gamma$ and Lyman- $\delta$ at 19:14:32 UT and Lyman-5 to Lyman continuum at 19:16:44 UT. The exposure time of each observation was $115 \mathrm{~s}$. All observed data were not binned during the digitization process in neither a spectral nor in a spatial direction. The dispersion is around $0.044 \AA$ /pixel. The signal-to-noise ratio for the centre of the Lyman- $\alpha$ line reaches 15 , for the centre of the Lyman- $\beta$ line it is around 8, for Lyman- $\gamma$ line-centre it is around 5 and for the centre of the higher Lyman lines it is below 2 .

Calibration and correction of the observed data was done by using standard Solar-Soft procedures (in the following order: dead-time correction, flatfield, local-gain correction and correction for geometrical distortion) to obtain intensities in counts per pixel per sampling interval. Finally, these intensities were converted into physical units using the radiometric calibration. The wavelength calibration was made according to Hansteen et al. (2000) without the correction for shifts of the spectral images on the detector (therefore the wavelength calibration is not absolute). However, the absolute wavelength calibration is not necessary for us because we do not take into account any macroscopic velocities in our modelling. The uncertainties of the intensity measurements depend on the number of photons registered in each pixel during the exposure; the more photons detected in the pixel the higher the signal-to-noise ratio. Using Poisson statistics, the relative error in each pixel is proportional to the reciprocal of the square root of the counts detected in the pixel during the sampling interval (exposure time). All above-mentioned procedures correct the data only to a certain extent. Some instrumental effects as well as additional uncertainties in the radiometric calibration curve for detector A can be still present. The uncertainties in the radiometric calibration curve can be around $15 \%$ for wavelengths between 540 and $1250 \AA$ and can rise up to $30 \%$ for higher wavelengths (Wilhelm et al. 1999).

The observations of Lyman- $\alpha$ used in our study are the only SUMER Lyman- $\alpha$ observations outside the attenuator. The line profiles of Lyman- $\alpha$ observed on SUMER by using the attenuator are unreliable (see Heinzel et al. 2001) and are thus not useful for the comparison with the synthetic profiles. With respect to the end of the lifetime of the SUMER detectors, observation of the intense Lyman- $\alpha$ line outside the attenuator is now allowed, making realistic Lyman- $\alpha$ profiles obtaining possible.

\subsection{Observational data}

Each observed spectrum consists of 120 spatial positions along the SUMER slit. From these only pixels 6 to 60 belong to the operational part of the SUMER detector (Fig. 1) and contain useful data. The profiles of each observed Lyman line exhibit rather strong variation along the slit from more intense reversed profiles on the first 30 pixels of the slit to lower emission profiles on another 30 pixels. Figure 2 shows the spectra of the Lyman- $\alpha$ to Lyman- $\delta$ lines from pixels 1 to 60 . Figure 3 shows the variation of the intensity, integrated over the spectral domain of each line, along the slit for Lyman- $\alpha$ to Lyman- $\delta$ lines. The values of the integrated intensity (Fig. 3) in comparison with the values of the integrated intensity from the disk (Heinzel et al. 2001, Table 3) clearly show that the observed data we use belong to the prominence. 

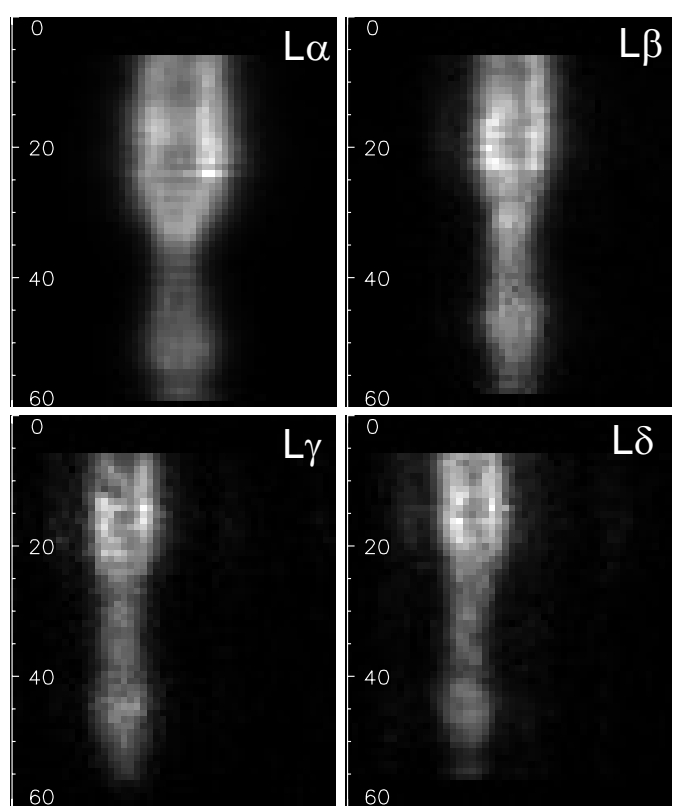

Fig. 2. SUMER spectra for Lyman- $\alpha$ to Lyman- $\delta$ lines from pixels 1 to 60 .

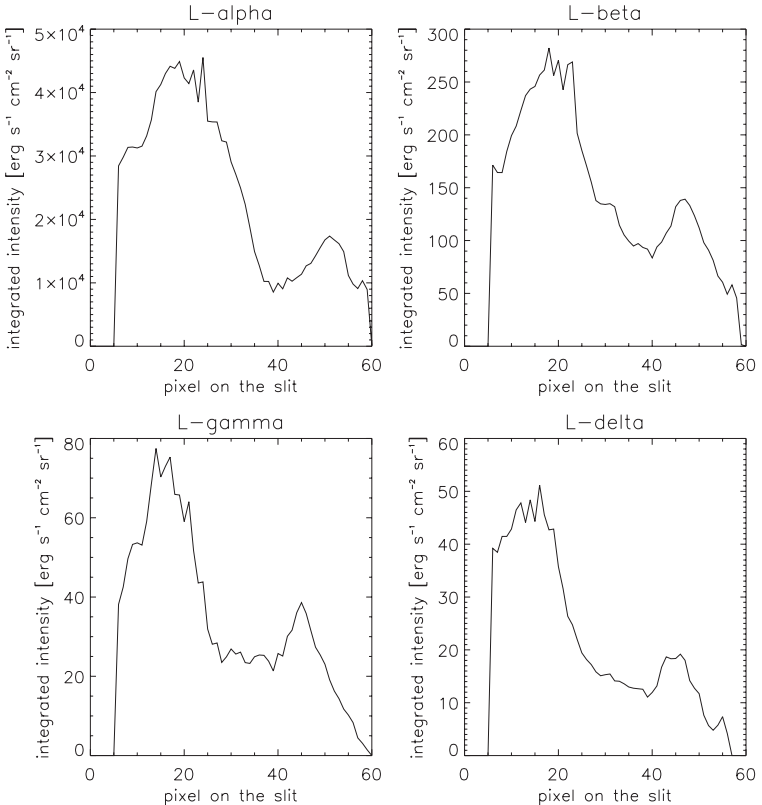

Fig. 3. The variation of the integrated intensity (over the spectral domain of each line) for Lyman- $\alpha$ to Lyman- $\delta$.

In addition, reversed profiles show strong asymmetries of their peaks most probably due to velocity gradients. For the analysis of the Lyman lines by comparison with our synthetic spectra it is necessary to use symmetrical profiles. Such profiles which are almost symmetrical, occur in our observational data in small clustered locations of up to 3 pixels close to each other. An averaging over such an area can help us to avoid errors due to the possible presence of velocity gradients while the spatial resolution remains on the same level as the model resolution. The clusters of pixels with symmetrical profiles for all of the observed Lyman lines, lie between pixel 13 and 18 along the slit as follows:

The Lyman- $\alpha$ profile represents the average over pixels 14 to 17 , Lyman- $\beta$ over pixels 13 and 14 , Lyman- $\gamma$ over 15 to 17 , Lyman- $\delta$ over 13 to 15 , the observed profile of the Lyman- 6

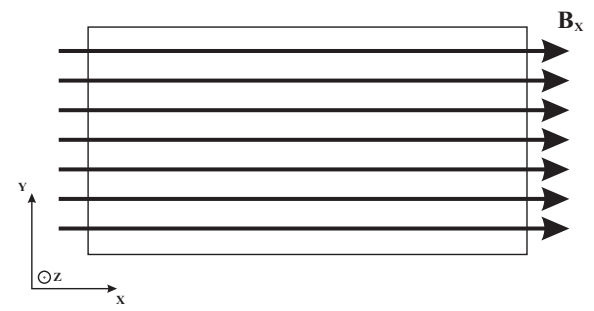

Fig. 4. Sketch of the $x-y$ horizontal plane with the outlined direction of the horizonal magnetic field $B_{x}$.

represents the profiles from pixel 15 and the Lyman-7 profile represents the average of pixels 15 and 16 . The observed data of the Lyman-5 line do not contain useful symmetrical profiles and therefore we cannot use them in this study. The observed profiles of the Lyman lines higher than Lyman-7 contain a noise level which is too high to be useful for our study.

\section{2D models of the prominence fine structure}

In order to obtain the synthetic spectra we use the 2D prominence fine structure models presented by Heinzel \& Anzer (2001). The prominence takes the form of a vertically infinite two-dimensional thread hanging in the a horizontal magnetic field. The variation of all quantities takes place in the $x-y$ horizontal plane (parallel to the solar surface) with the $z$-axis pointing upwards. The thread is uniform along the $z$-axis (Fig. 4). The mathematical formulation of the 2D MHS equilibrium for such fine structure threads is described by Eqs. (2) to (7) of Heinzel \& Anzer (2001). Generally, the magnetic field in prominences exhibits a strong shear component (e.g. Bommier et al. 1994). However since the fine structure threads are formed along the magnetic field independently of the shear of the magnetic field with respect to the prominence/filament axis, we do not take the shear component of the field into account. The input parameters for the MHS equilibrium are boundary (coronal) pressure $p_{0}$, the intensity of the magnetic field in the middle of the thread $B_{x}(0)$ and the maximum column mass $M_{0}$ which appears in the formula for $M(y)$ :

$M(y)=M_{0}\left(1-\left|\frac{y}{\delta}\right|^{\gamma_{3}}\right), \quad$ for $\quad|y| \leq \delta$,

where $2 \delta$ represents the width of the thread (dimension across the field lines) and exponent $\gamma_{3}$ is set to 2 in our models.

The empirically given temperature profile (Heinzel \& Anzer 2001) considers two different shapes of the PCTR. Along the field lines (along the length of the thread) the temperature gradually increases from the central coolest part of the prominence thread towards the boundary temperature. In contrast, across the field lines the temperature exhibits a very steep gradient towards the boundary, within a very thin PCTR layer. This is caused by strongly inhibited heat conduction across the magnetic field lines. The temperature profile can thus be expressed as

$T(m, y)=T_{\mathrm{cen}}(y)+\left[T_{\mathrm{tr}}-T_{\mathrm{cen}}(y)\right]\left\{1-4 \frac{m}{M(y)}\left[1-\frac{m}{M(y)}\right]\right\}^{\gamma_{1}}$,

where $T_{\text {tr }}$ represents the temperature at the boundary and the exponent $\gamma_{1}$ is properly chosen to describe the gradual increase of the temperature along the field lines. The column-mass coordinate $m$ characterizes the extension of the thread in the geometrical $x$ direction. $T_{\text {cen }}(y)$, is given by

$T_{\text {cen }}(y)=T_{\text {tr }}-\left(T_{\text {tr }}-T_{0}\right)\left(1-\left|\frac{y}{\delta}\right|^{\gamma_{2}}\right), \quad$ for $\quad|y| \leq \delta$. 
Here $T_{0}$ is the (minimum) central temperature and the exponent $\gamma_{2}$ is chosen to describe the steep temperature gradient across the field lines.

In the formulation of the MHS equilibrium and for the temperature profiles, we used the column-mass coordinate $m$ instead of $x$ which makes the equilibrium equations independent of the assumed temperature profile. The transformation to the $x$ coordinate requires the following integration

$x=\int_{M(y) / 2}^{m} \frac{1}{\rho} \mathrm{d} m^{\prime}$

at each value of the coordinate $y$, where $\rho$ is the density. The relation between $p$ and $\rho$ is given by the equation of state (Heinzel $\&$ Anzer 2001) and depends on the ionization-degree structure.

In order to solve the radiative transfer problem, we assume that the thread is uniformly irradiated from all sides by incident solar radiation. For solving the $2 \mathrm{D}$ radiative transfer we use the method based on the Accelerated Lambda Iteration (ALI) technique (Auer \& Paletou 1994) with the usage of the Short Characteristics (SC) method for obtaining the formal solution along individual rays. A detailed description of the method is given in Heinzel \& Anzer (2001). In order to obtain the higher members of the Lyman series we use a 12-level plus continuum hydrogen atom model. The partial redistribution for the Lyman$\alpha$ and Lyman- $\beta$ lines is treated as in Heinzel et al. (1987) and in Paletou (1995).

\section{Comparison of observed and synthetic spectra}

The two-dimensional prominence fine-structure thread models provide us with the possibility to see the thread from different directions and thus with different orientations of the magnetic field (and corresponding PCTRs) with respect to the line-ofsight. As suggested by Heinzel et al. (2001) and shown by Heinzel et al. (2005), synthetic Lyman-line profiles exhibit the self-reversal shape even for higher members of the Lyman series when seen across the field lines. On the other hand, the profiles of the Lyman lines higher than Lyman- $\alpha$ usually have unreversed emission shapes when seen along the field. This is caused by quite different structures of the PCTRs formed across and along the magnetic field. Our observed Lyman spectra show the reversed shapes even for higher lines and therefore we focus our study only on the line-of-sight perpendicular to the magnetic field.

\subsection{Resulting fine-structure thread models}

Given that we concentrate our investigation on the direction of the line-of-sight perpendicular to the magnetic field we see the prominence thread along its width (in our models the width is $1000 \mathrm{~km}$ along the $y$-direction) with two narrow PCTR regions with very steep gradients of the temperature. As was shown in Heinzel et al. (2005), the Lyman-line profiles show rather significant shape and intensity variations at different positions along the length of the prominence thread. Figure 5 shows the variation of the Lyman- $\alpha$ profiles (half of each line profile is displayed) emerging from different positions along the length of the thread (the position number is indicated on the $x$-axis, half of the thread is displayed where position 42 corresponds to the centre of the thread). Each of the line profiles is the average profile over three positions with the centre at the position of the resulting profile. Note that the distance between each of the positions is approximately $350 \mathrm{~km}$ and the pixel-size of the SUMER is roughly

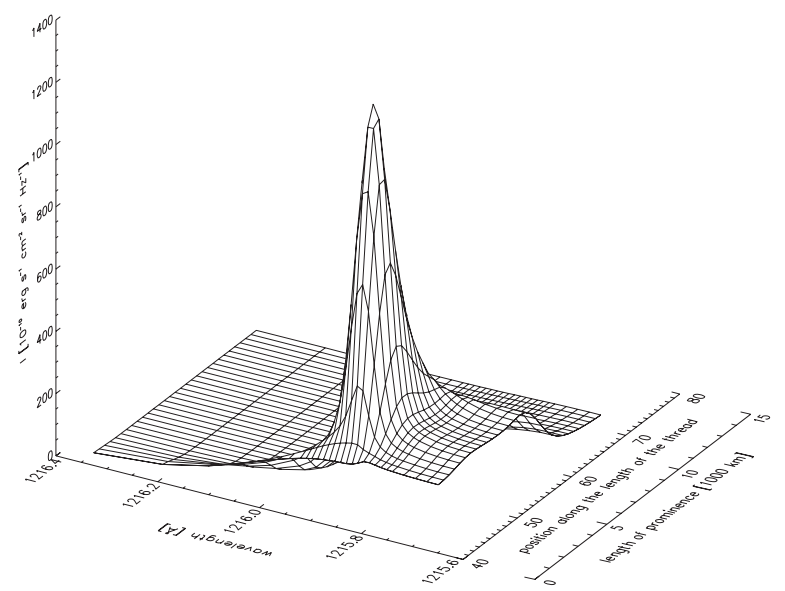

Fig. 5. Emergent Lyman- $\alpha$ intensity variation along the length of the prominence thread. The surface plot shows the half of the Lyman- $\alpha$ profile in different positions along the length of the half of the thread from its centre (centre corresponds to the position 42). Numbers on the $x$-axis indicate the position along the thread. The second $x$-axis shows the length of the prominence thread from its centre in $10^{3} \mathrm{~km}$.

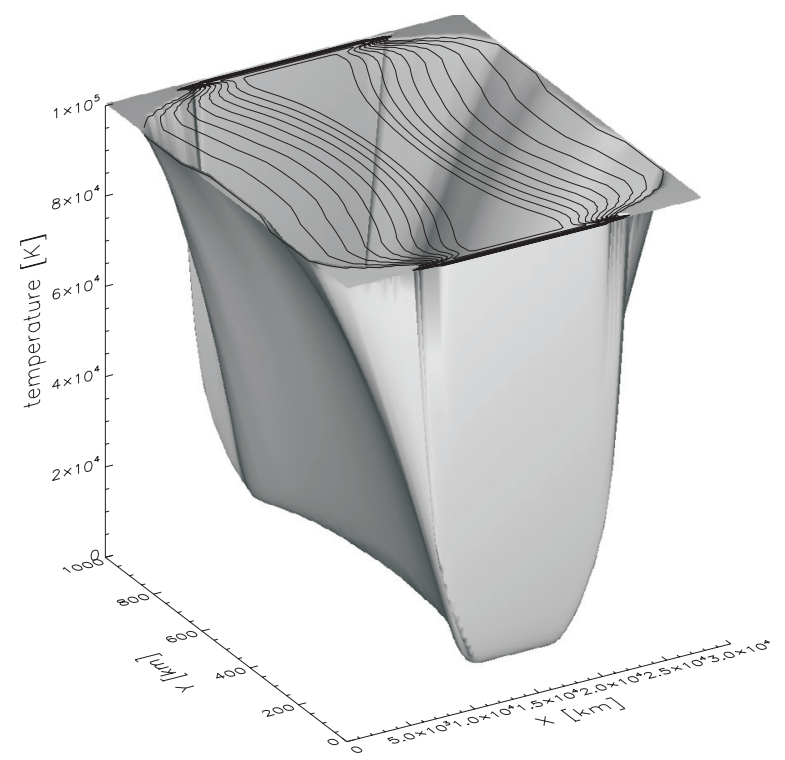

Fig. 6. Variation of the temperature for the resulting prominence thread model. Iso-contours of the temperature range from 10000 to $100000 \mathrm{~K}$ with steps of $10000 \mathrm{~K}$.

twice that size. The second $x$-axis in Fig. 5 shows the geometrical length of the thread from its centre. Thus the $x$-dimension of the thread is approximately $30000 \mathrm{~km}$ while the $y$-dimension is $1000 \mathrm{~km}$.

To obtain the model with the best agreement between the observed and synthetic Lyman-line profiles we used a trial-anderror method beginning with the grid of 18 models of Heinzel et al. (2005). By varying the input parameters (i.e. central temperature, central column mass, magnetic field intensity and boundary pressure), and comparing the synthetic profiles emerging from different positions along the length of the thread with the observed line profiles, we identified a model, which is, as far as we can say, unique in the sense that one cannot find any other model in the parameter space which will not lead to a similar agreement between observed and synthetic Lyman- $\alpha$ to Lyman- $\delta$ lines. However, it is possible to find a slightly better fit by small variations (in the order of few a percent) of the input parameters 

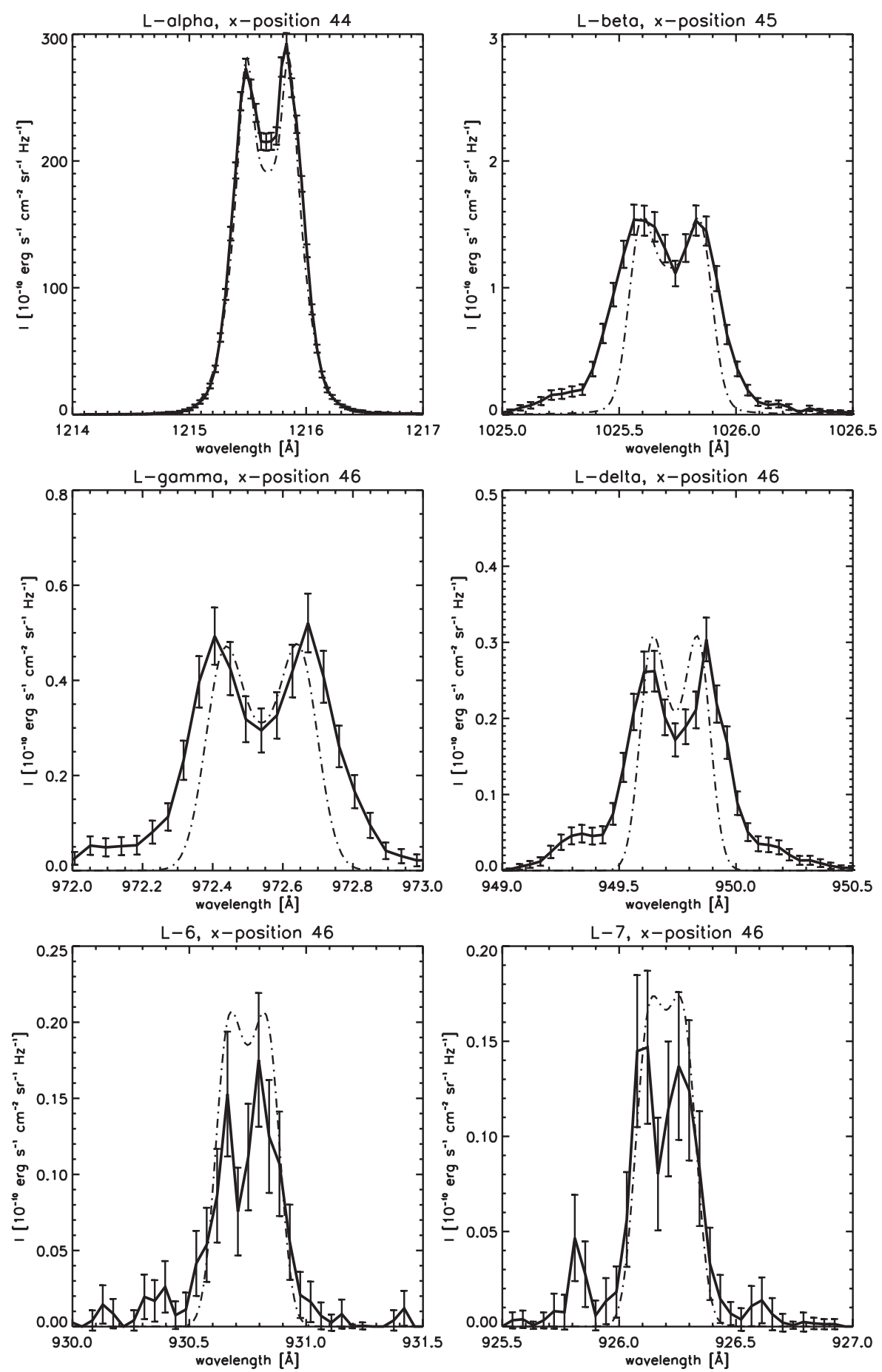

Fig. 7. Comparison between synthetic and observed spectral lines from Lyman- $\alpha$ to Lyman-7. Full lines with overplotted error bars represent the observed data. Dot-dashed lines represent the single-thread model synthetic profiles emerging from the indicated position along the thread.

around the values of the resulting model but such small differences in the shape of the line profiles do not have any influence on our conclusions.

The input parameter values of the resulting model are the central (minimum) temperature $T_{0}=7000 \mathrm{~K}$, maximum column mass in the centre of the thread $M_{0}=1.1 \times 10^{-4} \mathrm{~g} \mathrm{~cm}^{-2}$, horizontal field strength in the middle of the thread $B_{x}(0)=6$ Gauss

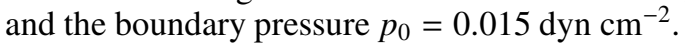

Figure 6 represents the variation of the temperature for the resulting prominence fine-structure thread. The iso-contours of the temperature (ranging from 10000 to $100000 \mathrm{~K}$ with steps of $10000 \mathrm{~K}$ ) show the very steep gradient of the temperature in the $y$-direction (across the magnetic field) and gradually increasing temperature along the $x$-direction (along the magnetic field). Note that in Fig. 6 the $x$-dimension of the thread is approximately $30000 \mathrm{~km}$ while the $y$-dimension is $1000 \mathrm{~km}$.

\subsection{Comparison of single-thread model spectra with observed profiles}

The comparison of the observed and synthetic Lyman- $\alpha$ to Lyman-7 lines is shown in Fig. 7, where the full lines with overplotted error bars represent the observed data and the dotdashed lines represent the synthetic profiles emerging from the indicated positions along the length of the thread. The observed Lyman- $\alpha$ line matches with the synthetic profile 
emerging from position 44, Lyman- $\beta$ with the profile from position 45 and Lyman- $\gamma$ and Lyman- $\delta$ with the profiles from position 46. Lyman-6 and Lyman-7 are not in agreement with any of the synthetic profiles, however the profiles emerging from position 46 have the closest similarity.

The shift in the positions along the thread from which the profiles are emerging with the best agreement with the observed ones cannot be explained by the time difference between the particular observations (approximately $2 \mathrm{~min}$ ) and it seems rather nonphysical. A possible explanation of this effect could be the introduction of an inclination between the line-of-sight and the surface of the thread. This has a minor effect on the Lyman- $\alpha$ line profile which, due to its large optical thickness, originates very close to the surface of the thread. However, the importance of this effect is rising with decreasing optical thickness and thus the higher members of the Lyman series are more affected. This can, in general, shift the emerging position of synthetic profiles back to the emerging position of the Lyman- $\alpha$ line. A more detailed description of this effect would need a very extended investigation which is not the purpose of the present study.

Another problem is the width of the synthetic spectral lines which is too small in comparison with the observed ones while the central parts of the lines are in good agreement. Better results can be obtained using the multi-thread modelling described below.

\subsection{Multi-thread modelling}

Our multi-thread modelling consists of a set of the 2D prominence fine-structure threads placed perpendicularly to the lineof-sight in random positions (Fig. 8). These threads are assumed to be identical. We do not consider any radiative interaction between the threads and thus the distance between them is not important for the results. The total multi-thread model intensity for a given wavelength at any position along the length of the first thread can be described by the formula

$I_{\text {total }}=I_{\mathrm{P}_{1}}+\exp \left(-\tau_{\mathrm{P}_{1}}\right) \times I_{\mathrm{P}_{2}}+\ldots+\exp \left(-\sum_{1}^{N-1} \tau_{\mathrm{P}_{i}}\right) \times I_{\mathrm{P}_{N}}$

where $I_{\mathrm{P}_{1}}$ represents the intensity from the given position on the first thread. The thread $i(i>1)$ is randomly shifted with respect to the first thread, $\mathrm{P}_{i}$ represents the position along the length of the thread $i$ which is on the same line-of-sight as the given position on the first thread and $I_{\mathrm{P}_{i}}$ represents the intensity emerging from position $\mathrm{P}_{i} \cdot \tau_{\mathrm{P}_{1}}$ is the optical depth at the given position along the first thread and $\tau_{\mathrm{P}_{i}}(i>1)$ represents the optical depth at the position $\mathrm{P}_{i}$ along the thread $i . N$ represents the total number of the threads. Note that in the 1D case the multi-thread fine structure is modeled by a series of parallel planes which are uniform along the length and thus have the same properties at every position $\left(\mathrm{P}_{i}\right)$ (e.g. Fontenla et al. 1996).

For our study we have considered the first plus 9 additional randomly placed threads each representing the same basic model which has been described previously. The random distributions of the threads produce different emerging profiles for different arrangements of the threads. Figure 9 shows the results of the multi-thread modelling for Lyman- $\alpha$ to Lyman-7 (except Lyman-5). The full lines with overplotted error bars represent the observed profiles. The gray full lines show the one hundred randomly arranged multi-thread models and the dot-dashed lines are the profiles emerging from the first thread only. The position along the first thread is marked in the title of each plot. The area within the gray profiles covers the range of all emerging profiles.

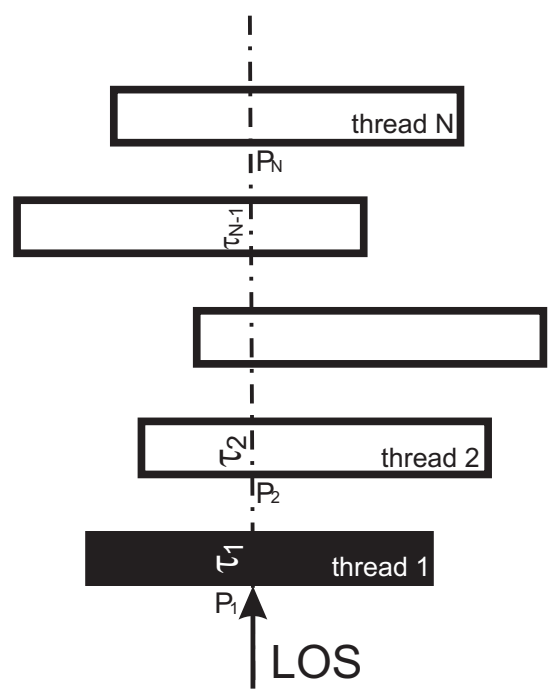

Fig. 8. Scheme of the model with randomly arranged threads. LOS is the line-of-sight. For other quantities see the text.

The Lyman- $\alpha$ line is not strongly affected by the multi-thread modelling and the line profile has almost the same shape as the profile obtained by the single-thread model (except for the slightly wider far wings). The same effect takes place for each position close to the centre of the thread (at position 42).

The shape of the Lyman- $\beta$ line is however significantly changing with different arrangements of the threads. Here we plot the profiles emerging from position 44 instead of position 45 which was shown in Fig. 7, because at position 44 the observed profile is within the probability region of the multi-thread model profiles. At position 45 the observed profile is on the lower edge of the probability area. The line-wings are significantly wider than the line-wings of the single-thread model. On the left side of the Lyman- $\beta$ line there is a blending line of He II (1025.27 $\AA$ ).

The effect of the multi-thread modelling on Lyman- $\gamma$ and Lyman- $\delta$ line profiles is the same as on the Lyman- $\beta$ line. It means that the profiles are significantly different from the singlethread profile with higher peaks and wider wings. The observed profiles are within the probability area at position 45 . The far wings are again blended by He II (972.12 $\AA$ and $949.36 \AA)$ on the left side of the Lyman lines and by O I (973.24 $\AA$ and 950.11 $\AA$ ) on the right side.

The Lyman-6 and Lyman-7 lines at position 44 show much better agreement for the multi-thread probability area than with the single-thread profiles emerging from position 46 .

\section{Discussion and conclusions}

The present study shows that prominence Lyman lines observed by the SUMER spectrograph can be better reproduced using multi-thread modelling than with single-thread models. As shown in Fig. 9 the width of the synthetic profiles is significantly larger and thus in better agreement than the width of the singlethread model. With multi-thread modelling we are able to reproduce even higher lines of the Lyman series which is not possible considering only a single prominence fine-structure thread. In addition, the shift of the emerging positions of the synthetic profiles with the best agreement with the observed ones is not as large as it is for the single-thread model. The modelling can be further improved by the introduction of the angle between the line-of-sight and the thread surface which can be different from 

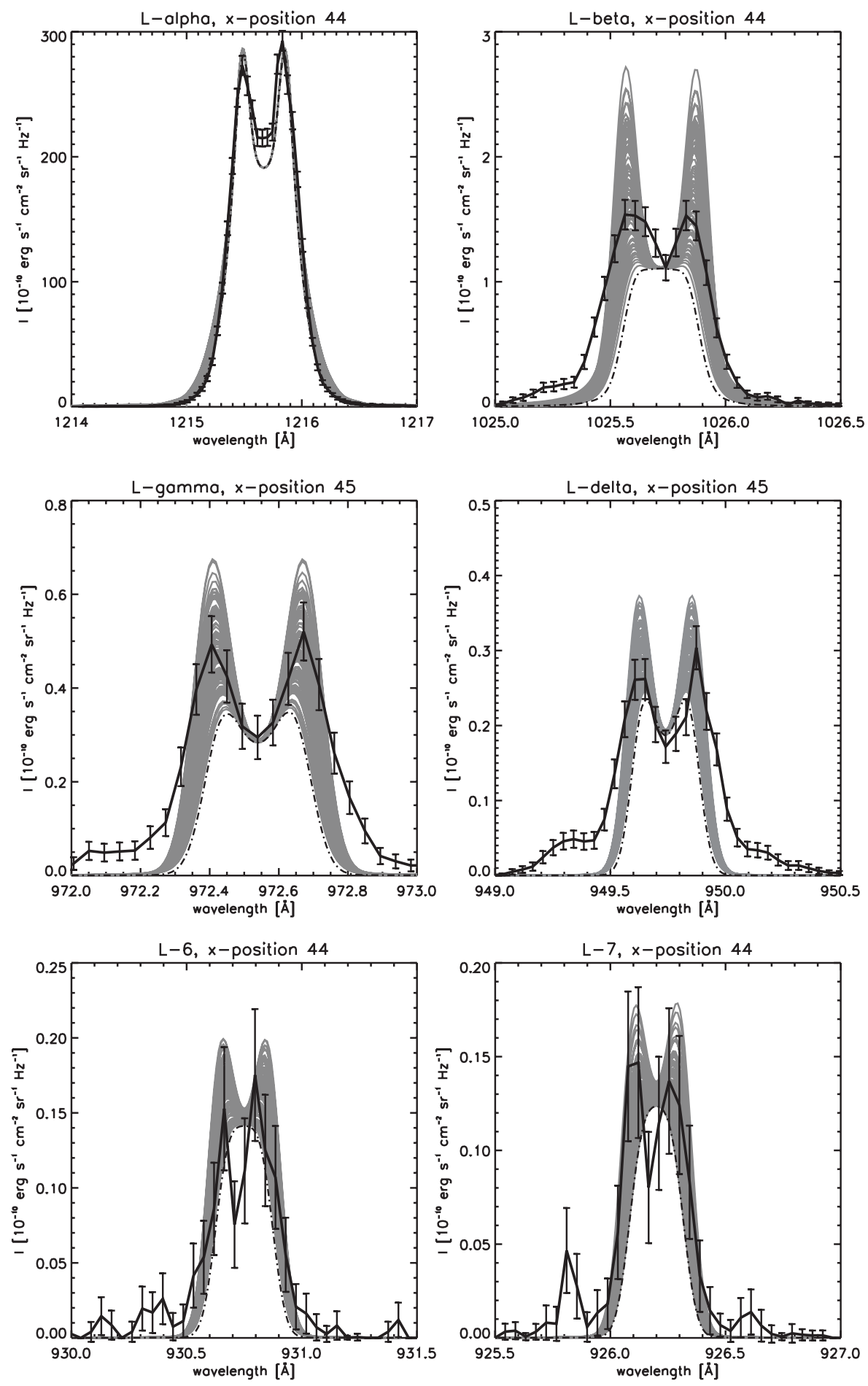

Fig. 9. Comparison between the synthetic and the observed spectral lines Lyman- $\alpha$ to Lyman-7. Full lines with overplotted error bars represent the observed profiles. Gray full lines belong to the one hundred randomly arranged multi-thread models and the dot-dashed lines are the profiles emerging from the first thread from the position which is marked in the title of each plot.

the perpendicular direction. The observations of the filaments on the disk show a number of the fine-structure thread-like features which are on the limb projected along the line-of-sight and thus further support the importance of the multi-thread modelling.

The multi-thread modelling with randomly arranged threads allows us to see the probable shape of the synthetic profiles and thus we are able to deduce the position along the thread with the emerging profile which gives the best agreement with the observations. In order to find the particular arrangement of the threads with the best match with the observed line profiles one has to perform an extensive investigation of all of the possible thread arrangements with different total numbers of threads. The total number of threads can be estimated by the value of the summed column mass of these threads along the line-of-sight. The total column mass of ten threads we have used in our study is of the order of $1.0 \times 10^{-4} \mathrm{~g} \mathrm{~cm}^{-2}$ which is in agreement with the values determined from observations by Anzer \& Heinzel (1998).

The Lyman- $\alpha$ line profile is not affected by multi-thread modelling (the resulting emerging profiles from multi-thread models are practically identical with the single-thread profile) and thus it is suitable for search for the best prominence finestructure thread model, which is afterwards used in the multithread modelling. 
The possibility to reproduce simultaneously all of the Lyman- $\alpha$ to Lyman-7 lines gives us good opportunity to determine in detail the physical properties of the prominence fine structure.

A more precise determination of the arrangements and the total number of the fine-structure threads requires an extended study of all possibilities of the arrangements of the threads in the multi-thread modelling as well as the inclusion of the radiative interaction between individual threads. We shall investigate this complex behaviour in our next paper.

Acknowledgements. S.G. and P.H. acknowledge the support from the MPA Garching; U.A. thanks for support from the Ondřejov Observatory. This work was done during S.G.'s stay at the MPA Garching supported by the EARA-EST programme and ESA-PECS project No. 98030. S.G. and P.S. acknowledge the support from the grant GA CR 205/07/1100. SOHO is a space mission of international cooperation between ESA and NASA.

\section{References}

Anzer, U., \& Heinzel, P. 1998, Sol. Phys., 179, 75

Auer, L. H., \& Paletou, F. 1994, A\&A, 285, 675

Fontenla, J. M., Rovira, M., Vial, J.-C., \& Gouttebroze, P. 1996, ApJ, 466, 496

Bommier, V., Landi degl'Innocenti, E., Leroy, J.-L., \& Sahal-Brechot, S. 1994, Sol. Phys., 154, 231
Gunár, S., Teriaca, L., Heinzel, P., \& Schuehle, U. 2006, in SOHO 17 - 10 Years of SOHO and Beyond, ed. H. Lacoste, ESA SP-617

Gunár, S., Heinzel, P., \& Anzer, U. 2007a, A\&A, 463, 737

Gunár, S., Heinzel, P., Schmieder, B., \& Anzer, U. 2007b, in The Physics of Chromospheric Plasmas, ed. P. Heinzel, I. Dorotovic, \& R. J. Rutten, ASP, 368,317

Hansteen, V. H., Betta, R., \& Carlsson, M. 2000, A\&A, 360, 742

Harrison, R. A., Sawyer, E. C., Carter, M. K., et al. 1995, Sol. Phys., 162, 233

Heasley, J. N., \& Mihalas, D. 1976, ApJ, 205, 273

Heinzel, P. 2007, in The Physics of Chromospheric Plasmas, ed. P. Heinzel, I. Dorotovic, \& R. J. Rutten, ASP, 368, 271

Heinzel, P., \& Anzer, U. 2001, A\&A, 375, 1082

Heinzel, P., \& Anzer, U. 2005, in Solar Magnetic Phenomena, ed. A. Hanslmeier, A. Veronig, \& M. Messerotti, Astron. Astrophys. Space Sci. Lib., 320 (Dordrecht: Springer), 115

Heinzel, P., Gouttebroze, P., \& Vial, J.-C. 1987, A\&A, 183, 351

Heinzel, P., Anzer, U., \& Gunár, S. 2005, A\&A, 442, 331

Heinzel, P., Schmieder, B., \& Vial, J. C. 2006, in SOHO 17 - 10 Years of SOHO and Beyond, ed. H. Lacoste, ESA SP-617

Heinzel, P., Schmieder, B., Vial, J. C., \& Kotrč, P. 2001, A\&A, 370, 281

Kippenhahn, R., \& Schlüter, A. 1957, Z. Astrophys., 43, 36

Labrosse, N., \& Gouttebroze, P. 2004, ApJ, 617, 614

Paletou, F. 1995, A\&A, 302, 587

Patsourakos, S., \& Vial, J.-C. 2002, Sol. Phys., 208, 253

Schmieder, B., Tziotziou, K., \& Heinzel, P. 2003, A\&A, 401, 361

Schmieder, S., Gunár, S., Heinzel, P., \& Anzer, U. 2007, Sol. Phys., 241, 53

Schmieder, B., Heinzel, P., Kucera, T., \& Vial, J.-C. 1998, Sol. Phys., 181, 309

Schmieder, B., Heinzel, P., Vial, J.-C., \& Rudawy, P. 1999, Sol. Phys., 189, 109

Wilhelm, K., Lemaire, P., Curdt, W., et al. 1995, Sol. Phys., 170, 75

Wilhelm, K., Woods, T. N., Schuehle, U., et al. 1999, A\&A, 352, 321 